\title{
Consumer networks and firm reputation: A first experimental investigation
}

\author{
STEFFEN HUCK, GABRIELE K. LÜNSER and JEAN-ROBERT TYRAN ${ }^{*}$
}

November 2007

\begin{abstract}
Arguing that consumers are the carriers of firms' reputations, we examine the role of consumer networks for trust in markets that suffer from moral hazard. When consumers are embedded in a network, they can exchange information with their neighbors about their private experiences with different sellers. We find that such information exchange fosters firms' incentives for reputation building and, thus, enhances trust and efficiency in markets. This efficiency-enhancing effect is already achieved with a rather low level of network density.
\end{abstract}

\section{Keywords}

Trust; Consumer networks; Moral hazard; Information conditions; Reputation

\section{JEL Classification Codes}

C72; C92; D40; L14

\footnotetext{
*Huck \& Lünser: Dept. of Economics and ELSE, University College London, Gower St, London WC1E 6BT, emails.huck@ucl.ac.uk / g.luenser@ucl.ac.uk; Tyran: Dept. of Economics, University of Copenhagen, and CEPR, London jean-robert.tyran@econ.ku.dk. Huck and Lünser acknowledge financial support from the Economic and Social Research Council (UK) via ELSE and an additional grant on "Trust and competition". Huck is also grateful for additional funding by the Leverhulme Trust. Tyran acknowledges support by FSE, Denmark.
} 


\section{Introduction}

A firm's reputation is based on the information its customers hold about its past performance. Such information may simply result from own past experience but more often than not it will actually stem from information exchange-from what consumers have heard from others. In fact, most brand reputation appears to be based on such word of mouth. In many cases, such as luxury cars or high-end stereos, consumers who know about a product's high quality far outnumber those who have actually tested it. Consequently, the way consumers exchange information matters for firms' reputation and, ultimately, for market outcomes. Consumer networks are the carrier of firms' reputations.

In this paper we use a simple experiment to shed some light on how the structure of consumer networks affects the performance of markets that suffer from moral hazard. Firms sell experience goods of unknown quality. Quality may be either good or bad. Buyers are matched to sellers and decide whether or not they want to buy a single unit of the good. When making this decision, buyers are provided with some information about the seller's past track record. The amount of information they are provided with depends on the underlying network structure of the buyer population. We consider four treatments: (NO) an anonymous one-shot benchmark where firms have no labels and reputation building is excluded by design; (DEGENERATE) a degenerate network without information exchange such that each buyer only knows his own experience; (PARTIAL) a partial network where buyers are located on a circle and everybody knows their own experience and that of their right-hand neighbor; (FULL) a full network where everybody has access to the entire history of all buyers and, due to one-to-one matching, the entire history of all sellers.

We find that markets fail completely when interaction is anonymous and one-shot. Without identification of firms there is no reputation building and market failure is almost complete. Only $5 \%$ of all matches result in mutually beneficial trade. Once there is identification that does allow reputation building market performance is vastly enhanced. In treatment DEGENERATE where all buyers just remember their own experiences the number of mutually beneficial trades almost quadruples compared to the anonymous benchmark. Buyers trust more often and sellers offer vastly better quality (see also Bolton et al. 2004, Bohnet and Huck 2004, Bohnet et al. 2005, or Bracht and Feltovich 2007). 
Comparing the different network treatments we then establish this note's main result-that market performance is increasing in network density. This increase in efficiency has two causes. Moving from DEGENERATE to PARTIAL we find that buyers are significantly more likely to trust sellers (that is, demand increases) while the average quality of traded goods stays constant. Moving from PARTIAL to FULL we find that, while demand stays constant, there is a significant rise in average quality.

\section{Experimental design and procedures}

To study how network density influences market performance we consider a binary-choice trust game with two players ${ }^{1}$ as shown in Figure 1. Here, the buyer (first mover) decides whether or not to buy an experience good and the seller (second mover) whether or not to deliver good quality. The chosen payoffs reflect a conflict of interest. While the buyer always prefers good over bad quality $(30>5)$, the seller has a strict preference to sell bad quality (50 $>25)$. In addition, the buyer prefers not to buy over getting bad quality $(20>5)$ and the seller would rather provide good quality than not selling at all $(25>15)$. Assuming that subjects maximize their own monetary income, the game has a unique Nash equilibrium in which the buyer does not buy and the seller provides bad quality if someone shops from him.

Figure 1: The trust game

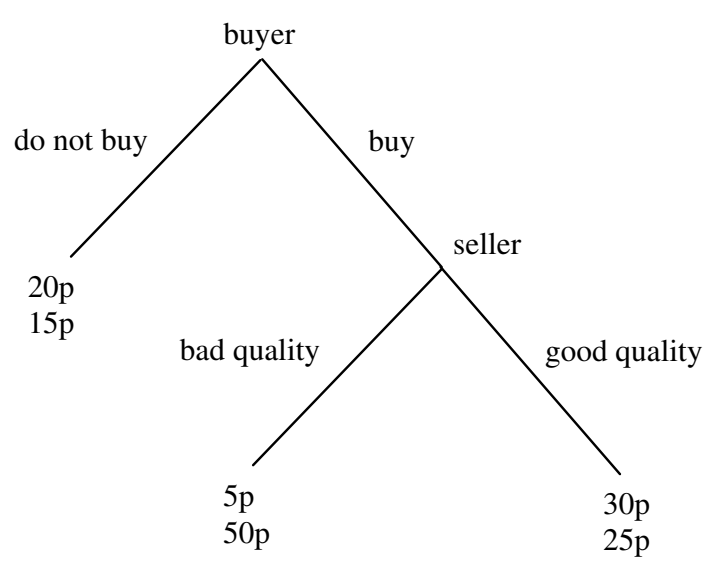

\footnotetext{
${ }^{1}$ In the experiment we adopt neutral language, e.g. a buyer is labeled A; seller - B; do not buy - X; buy - Y; etc. See Appendix for instructions.
} 
Our four treatments vary in their network density as follows:

- In NO, the baseline treatment, neither buyers nor sellers have any information about the past. This is the only treatment without identification of sellers making interaction completely anonymous.

- Introducing labeling of sellers, in DEGENERATE all buyers know from whom they shopped in the past. Thus, sellers now have to start thinking about direct reputation they establish with buyers.

- Increasing network density, in PARTIAL all buyers not only know their own experience but, additionally, also know the experience of one other buyer in the market. Thus, sellers may be concerned with indirect reputation effects which are mediated through consumers' network relation.

- Finally, in FULL we make the entire history of all sellers available to everybody. As already shown in Bohnet et al. (2005) this is the treatment that achieves the best market outcomes that the provision of feedback about sellers' past can achieve on its own. ${ }^{2}$

In each treatment, subjects play this game for 30 periods. Each matching group consists of four buyers and four sellers who keep their roles throughout the experiment. At the beginning of each period subjects are randomly rematched into pairs. For each treatment we have data of six independent observations collected with z-Tree (Fischbacher (2007)) at the University of London. Altogether, 192 subjects participated in the experiment which lasted on average less than an hour. Payoffs per period were exactly as shown in Figure 1 (with $p=$ pence). Average earnings were $£ 11.28$ (including a $£ 5$ show-up fee).

During the experiment, sellers' track records were displayed in the left part of the screen using a simple graphical representation. It showed four columns consisting of 30 hashes (\#) each column representing one seller and each row representing one period. Initially, all hashes were white. Then, after each period, hashes representing this period changed their color. They turned black if the seller had not to make a decision because his buyer did not buy from him. They turned green if the seller provided good quality of the good. They turned red if the seller exploited the buyer's trust and only provided bad quality. Additionally, in

\footnotetext{
${ }^{2}$ This treatment reflects a network structure where buyers and sellers are linked in a way such that everybody has full information.
} 
DEGENERATE and PARTIAL, where buyers did not receive all information about a market, hashes turned grey in the case where a buyer did not receive any information about a particular seller. Note that in the treatment NO there was no such graphical representation at all as sellers did not have labels. This easy and obvious color coding was chosen in order to make the quite complex history information easy to read. (Screenshots are shown in the Appendix.)

\section{Results}

In reporting the results we focus on three key variables: the average demand (or trust rate), that is, the share of first movers that purchased the experienced good; the average traded quality (or honor rate), that is, the share of second movers providing good quality (conditional on first movers having bought the experience good); and the share of mutually beneficial trades (or performance rate), that is the share of matches that resulted in (buy, good quality), our efficiency measure.

Table 1 reports these averages (and standard deviations) for the four different treatments and shows test statistics for a number of binary treatment comparisons (where the unit of observation is always one entire matching group).

In NO, where there are no incentives for reputation building, the trust and honor rate are very low and, as a consequence, so is the performance rate. Only in 5\% of all matches do subjects reach the mutually beneficial trade outcome. Introducing labeling of sellers in DEGENERATE increases the trust rate by half and more than triples the honor rate. This is not surprising: When customers can identify firms average quality improves drastically. As a result the number of mutually beneficial trades is nearly quadrupled.

Increasing network density in PARTIAL, by allowing each buyer not only to know his own experience but also the experience of a neighbor, improves market performance even further. However, this change is solely due to more trust in the market, average quality is statistically not distinguishable from average quality in DEGENERATE. Finally, when moving from PARTIAL to FULL we observe a further increase in the performance rate. This time the improvement is due to higher average quality while demand stays constant. 
Table 1: Overview of results

\begin{tabular}{lccc}
\hline \hline & trust rate & honor rate & performance rate \\
\cline { 2 - 4 } NO & 0.21 & 0.19 & 0.05 \\
& $(0.16)$ & $(0.11)$ & $(0.05)$ \\
DEGENERATE & 0.31 & 0.59 & 0.19 \\
PARTIAL & $(0.14)$ & $(0.15)$ & $(0.12)$ \\
& 0.44 & 0.48 & 0.23 \\
FULL & $(0.17)$ & $(0.16)$ & $(0.14)$ \\
& 0.43 & 0.62 & 0.29 \\
NO-DEGENERATE* & $(0.15)$ & $(0.23)$ & $(0.18)$ \\
DEGENERATE-PARTIAL* & \multicolumn{3}{c}{ adjacent treatment effects' } \\
PARTIAL-FULL* & $p=0.168$ & $p=0.002$ & $p=0.005$ \\
& $p=0.064$ & $p=0.168$ & $p=0.344$ \\
NO-FULL* & $p=0.468$ & $p=0.100$ & $p=0.235$ \\
NO-DEGENERATE- & \multicolumn{3}{c}{ further treatment effects } \\
PARTIAL-FULL & $p=0.027$ & $p=0.005$ & $p=0.008$ \\
\hline \hline
\end{tabular}

Standard deviations are given in parentheses. Treatment effects are tested by one-tailed Mann-Whitney U-tests $\left(^{*}\right)$ and Jonckheere-Terpstra tests $\left(^{\#}\right)$ respectively.

Our design gradually increases the amount of feedback information from treatment to treatment. As a consequence, several observed treatment differences of neighboring treatments are sometimes insignificant (see Table 1). However, we observe a continuous improvement in market performance due to higher network density. The Jonckheere-Terpstra test shows that there is an ordering for all three key variables (trust rate, honor rate and performance rate) of the treatments according to the network's density. ${ }^{3}$

\section{Conclusions}

In this note we have shown that efficiency in a market for experience goods increases in the density of an underlying consumer network that allows consumers to exchange information

\footnotetext{
${ }^{3}$ The Jonckheere-Terpstra test is a non-parametric test for ordered differences among classes. The alternative hypothesis assumes a certain ordering of the medians of $k$ statistically independent samples. All average values each of a statistically independent observation from a treatment with the same network density - are assigned to one class.
} 
about their private experience with different sellers. To the best of our knowledge this is the first study that provides such controlled experimental evidence in economics. We find that market performance is increasing in network density. While demand peaks with partial networks (where consumers just learn their own outcomes and those from one neighbor), average quality reaches its peak only in the full network where everybody has access to the entire history of all sellers. While such a full network represents a rather ideal case (perhaps only of real-world relevance on online trading platforms) it does help to benchmark the other outcomes. A partial consumer network with just 33\% of the full network's density reaches $80 \%$ of the ideal's efficiency. This result highlights the benefits of some minimal social cohesion. Informal information exchange with just a few neighbors may go a long way.

Our results should also be encouraging for future research on informal information exchange in networks. There are several interesting avenues of which we want to highlight two. In larger groups, it would be possible to reduce the network density further. How minimal can the density become before breakdown occurs? Secondly, what if information from others is not free, i.e., if links to neighbors need to be created and maintained? Our note suggests that it could be very exciting to study such endogenous formation of consumer networks in the laboratory. ${ }^{4}$

\section{References}

Bohnet, Iris; Harmgart, Heike; Huck, Steffen and Tyran, Jean-Robert (2005): Learning Trust, Journal of the European Economic Association 3, 322-329.

Bohnet, Iris and Huck, Steffen (2004): Repetition and Reputation: Implications for Trust and Trustworthiness when Institutions Change, American Economic Review 94, 362-366.

Bolton, Gary; Katok, Elena and Ockenfels, Axel (2004): How Effective are Electronic Reputation Mechanisms? An Experimental Investigation, Management Science 50, 1587-1602.

Bracht, Juergen and Feltovich, Nick (2007): An Experimental Study of Information Mechanisms in the Trust Game: Effects of Observation and Cheap Talk, Working paper, University of Aberdeen Business School and University of Houston.

Falk, Armin and Kosfeld, Michael (2003): It's All about Connections: Evidence on Network Formation, IEW Working Paper No. 146.

\footnotetext{
${ }^{4}$ For experiments on network formation with exogenous benefit structures see, for example, Falk and Kosfeld 2003 or Kosfeld's (2004) survey. In a setup like ours the gains of forming a link would endogenously depend on firms' reactions to the additional information flow.
} 
Fischbacher, Urs (2007): z-Tree: Zurich Toolbox for Ready-made Economic Experiments, Experimental Economics 10, 171-178.

Kosfeld, Michael (2004): Economic Networks in the Laboratory: A Survey, Review of Network Economics 3, 20-41. 


\section{Instructions (treatment PARTIAL)}

Welcome to our experiment!

Please read these instructions carefully! Do not speak to your neighbours and keep quiet during the entire experiment! In case you have a question raise your hand! We will then come to you.

In this experiment you will repeatedly make decisions. Doing this you can earn money. How much you earn depends on your decisions and on the decisions of other participants. All participants receive the same instructions.

All participants stay anonymous to the experimenter and also to other participants.

In the experimental situation there are two agents called $A$ and $B$, respectively. In each round every $A$ will be randomly matched with a B. Altogether there are eight participants. All participants are randomly assigned a role $(A$ or $B)$ at the beginning of the experiment and roles will be kept throughout the experiment. There will be four A-participants (A1, A2, A3, and A4) and four B-participants (B1, B2, B3, and B4).

At the beginning of each round each A-participant is randomly matched with a B-participant by the computer. That is, in each period the computer randomly chooses one of the Aparticipants and one of the B-participants who interact with each other in this period. This process is repeated in the next period. Therefore, you may meet with the same participant in two consecutive periods (in one out of four cases) or meet a different participant in the next period (in three out of four cases). In any case A-participants are told with whom they have been matched, i.e., A-participants will learn whether they have been matched with B1, B2, B3, or B4. (Of course, they will never learn which person in the room is behind any of these labels.) B-participants will NOT learn with whom they have been matched.

Once participants have been matched, it is A's turn to make a decision. More specifically, A has to choose between option X and option Y. If he picks option X, A will earn $20 \mathrm{p}$ and $B$ will earn $15 \mathrm{p}$. If he picks option $Y$, the payoffs depend on B's choice who has to decide whether he wants to go ,left" or „right". If he decides to pick ,left", A will earn $5 p$ and B will earn $50 \mathrm{p}$. If he decides to pick ,right“", A will earn $30 \mathrm{p}$ and B will earn $25 \mathrm{p}$.

These rules are illustrated in the following „tree“:

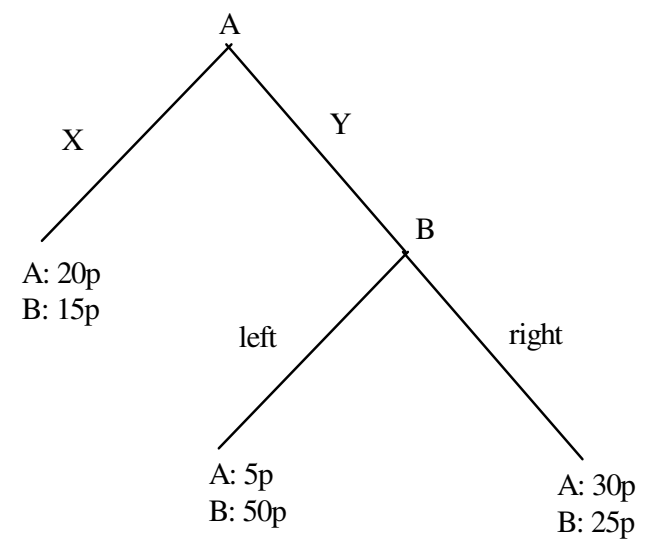


The experiment consists of 30 rounds. All participants keep the role and the number assigned to them throughout the experiment. After each round you will be informed about what has happened and you will be reminded of your earnings and your total earnings so far.

Moreover, A-participants can keep track of some of the history of B-participants. There will be a screen depicting the history of B-participants. For each round and each B there will be a coloured little \#. A black \# indicates that in this particular round the B-participant had nothing to decide because the A he had been matched with picked option X. A red \# indicates that B picked "left". A green \# indicates that B picked "right". Finally, a grey \# simply indicates that no information is available.

In each round each A-participant obviously learns what the B-participant did that he had been matched with. And this will be reflected by a coloured little \# in the above mentioned screen. Moreover, each A will be informed about one additional B in each round. In particular, A1 will also be informed about the B-participant that A2 had been matched with, A2 about the Bparticipant that A3 had been matched with, A3 about the B-participant that A4 had been matched with, and A4 about the B-participant that A1 had been matched with.

Accordingly, for each round A's will see for two B's either a black, green, or red \# and for two others grey \#'s. Or to put it differently, each round the behaviour of each B will not only be observed by the A he had been matched with but also by one other A.

These are the rules. You can trust us that everything will happen exactly according to these rules. Take your time going over these instructions again. And feel free to ask questions. But don't shout! Simply raise your hand.

Your total earnings that equal the sum of your individual earnings in the 30 rounds (plus your show-up fee of $£ 5$ ) will be paid to you right after the experiment in cash.

You have role $\mathbf{A}$. 
Screenshots (first mover)

NO

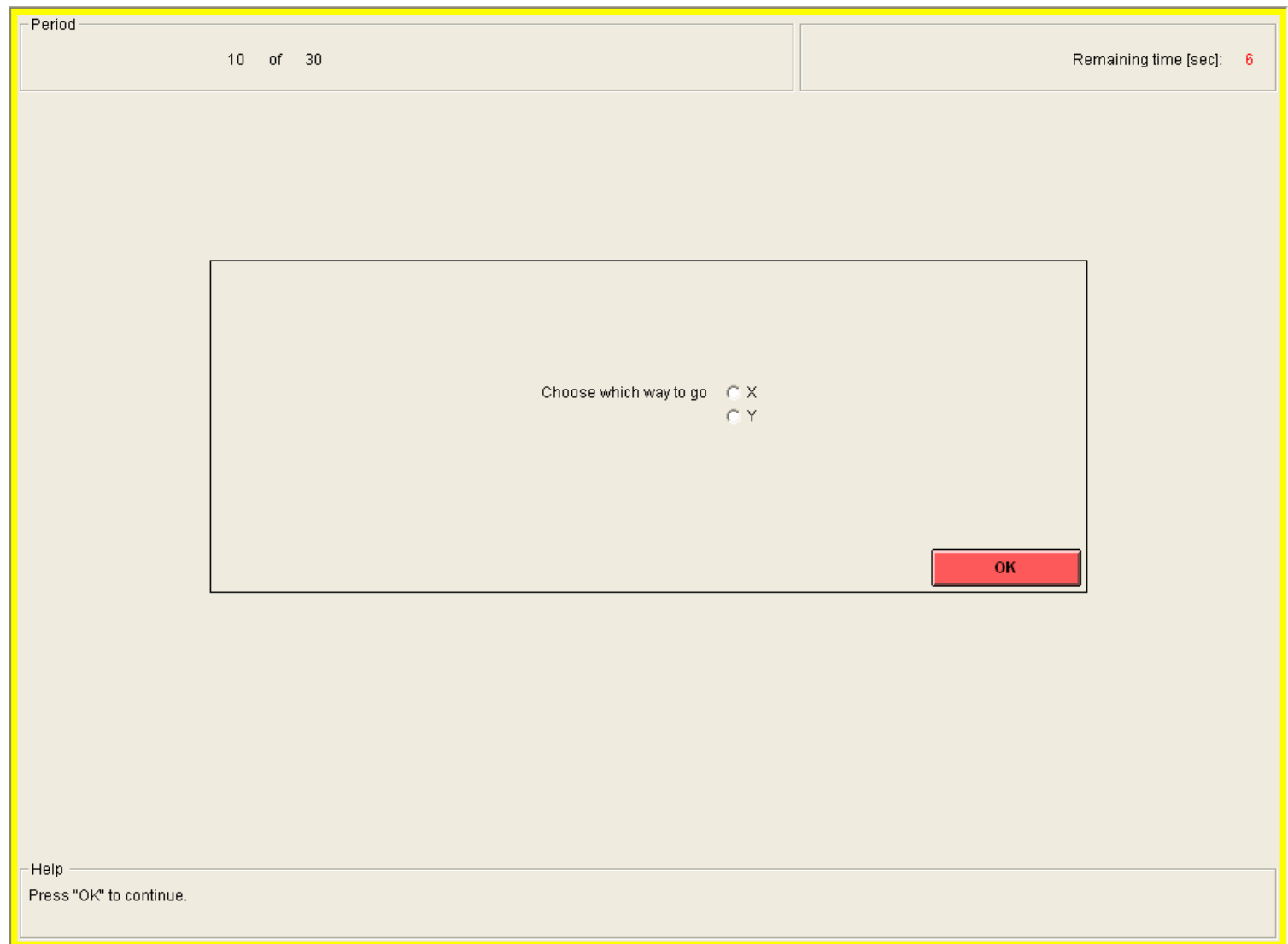

DEGENERATE

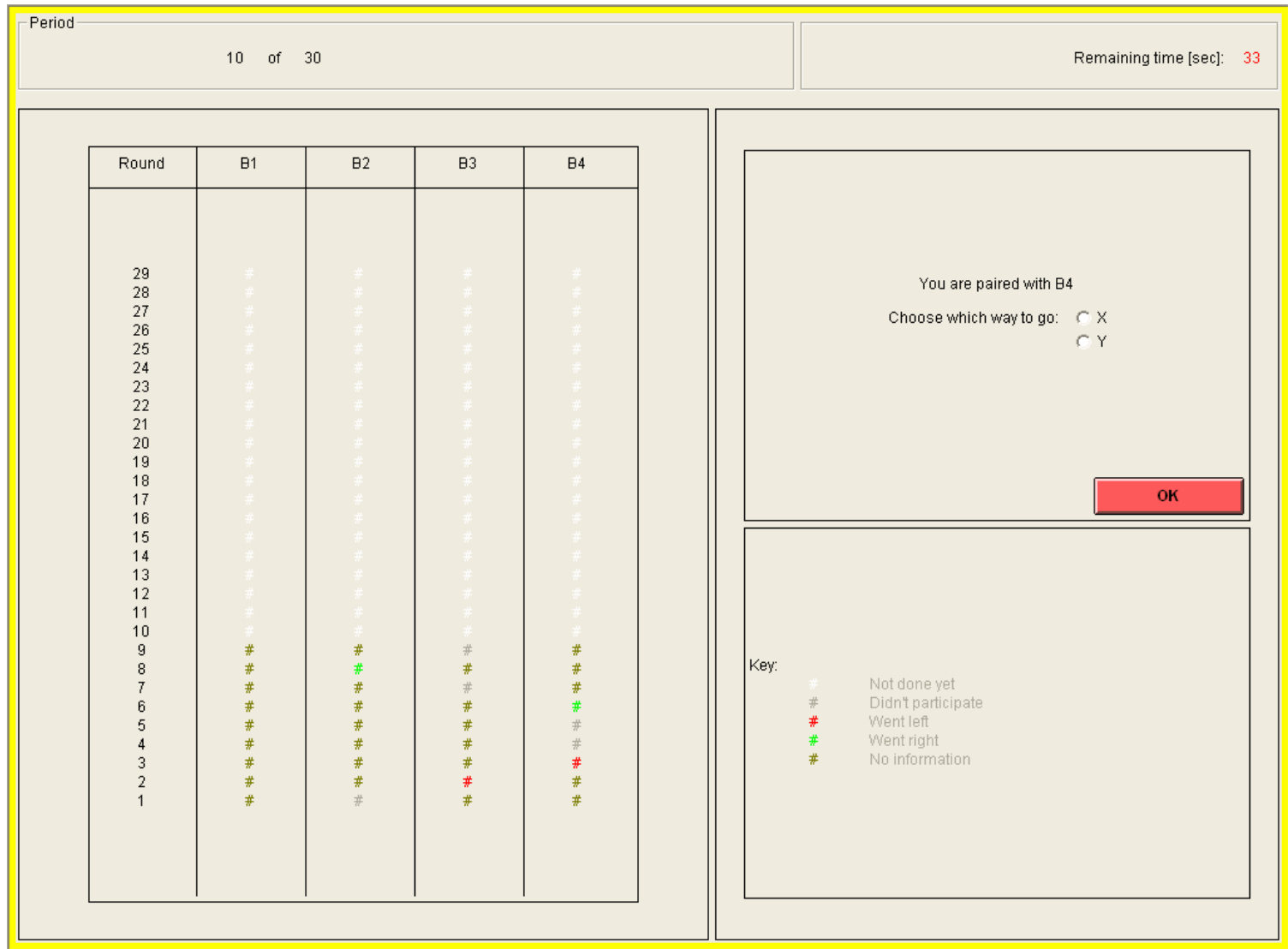


PARTIAL

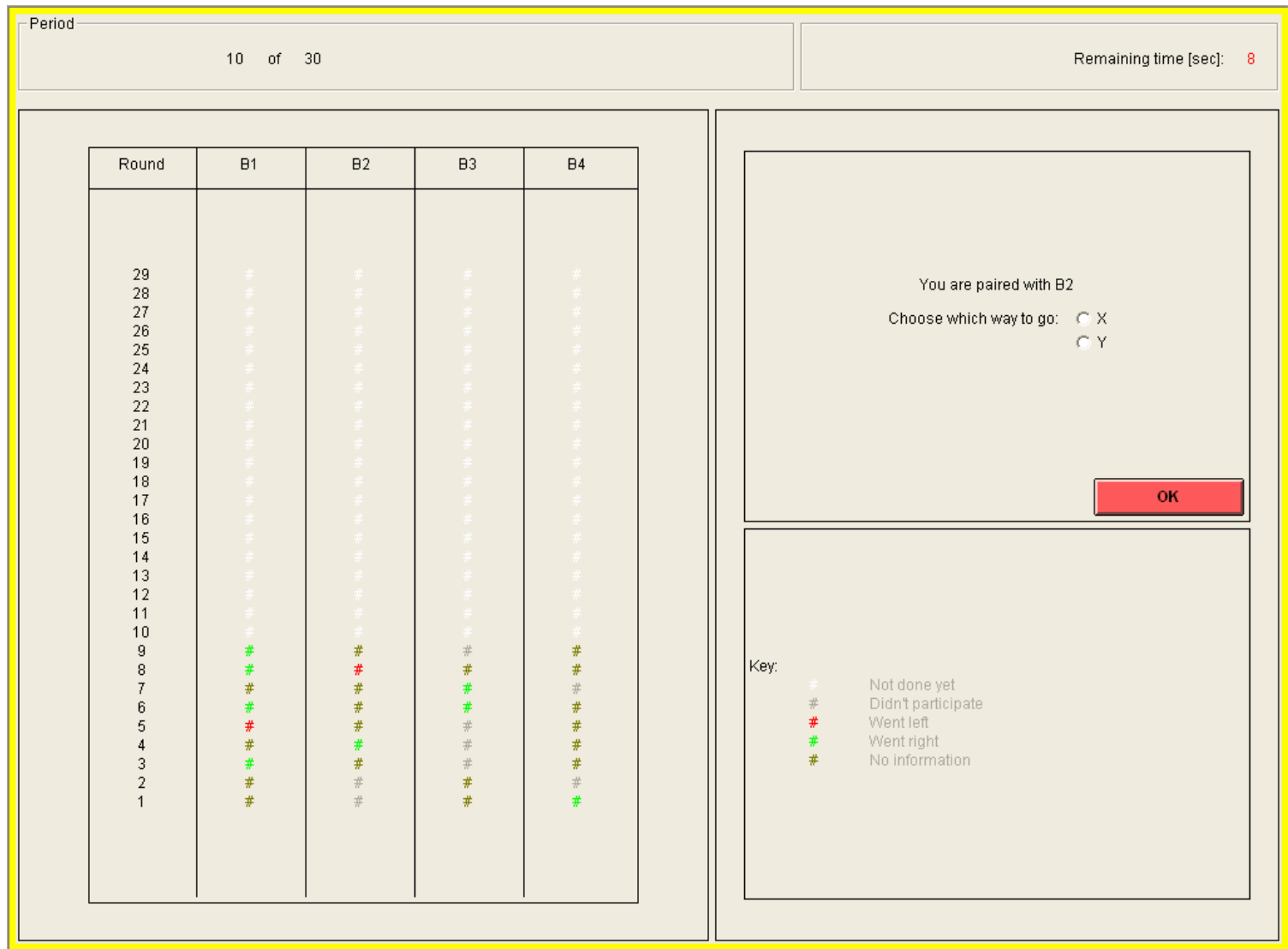

FULL

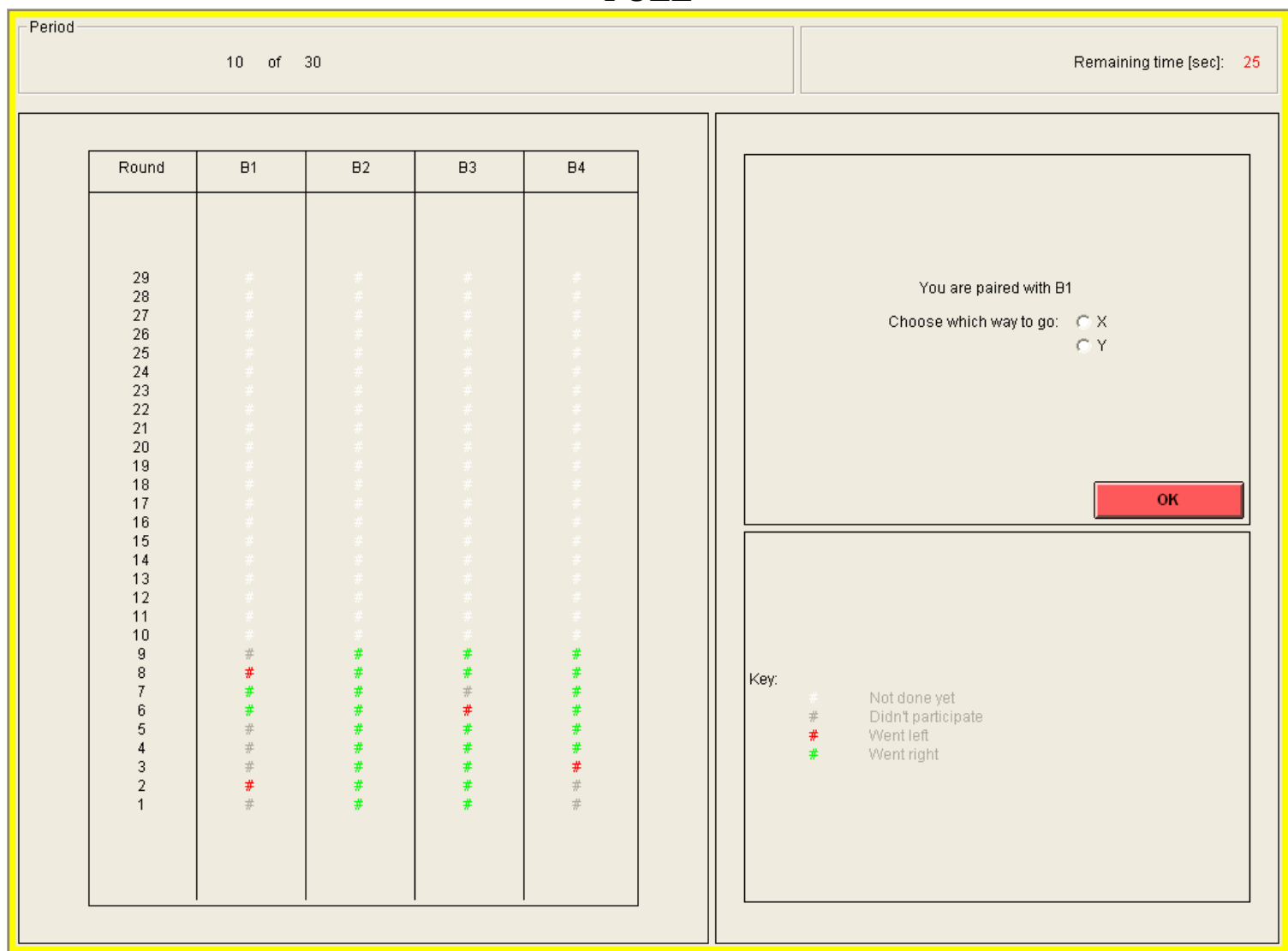

